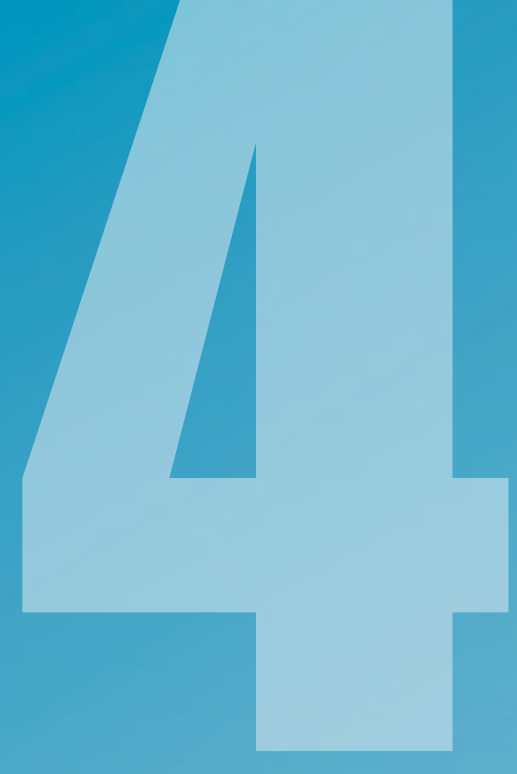

ÁMBITOS

REVISTA

INTERNACIONAL

DE COMUNICACIÓN

$N^{\circ} 46$

EDICIÓN OTOÑO

2019

ISSN: 1139-1979

E-ISSN: 1988-5733

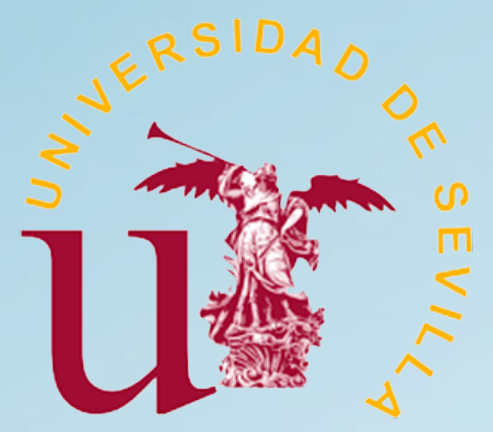




\section{ÍNDICE}

MONOGRAFICOS MONOGRAPHS

Presentación Monográfico. Comunicación emergente. Experiencias para el cambio social

Dra. Nereida López Vidales

Los formatos de televisión más consumidos por los jóvenes: telerrealidad y empoderamiento de la audiencia

Most consumed televisión formats by young people: real TV and the empowerment of the audience Nereida López Vidales, Leire Gómez Rubio, Elena Medina de la Viña

Nuevas herramientas, viejas costumbres El Contenido Generado por los Usuarios sobre el cambio climático en YouTube

New tools, old habits User Generated Content about climate change on YouTube

David Vicente Torrico

Tratamiento periodístico de personas LGTBIQ+ refugiadas: estudio de caso sobre Pride Barcelona 2018

Journalistic treatment of LGTBIQ+ refugees: case study on Pride Barcelona 2018

Hadriel Theodoro, Amparo Huertas Bailén

Tiempo Muerto, estudio de caso de un proyecto transmediático para la consecución de competencias universitarias

Tiempo Muerto, case study of a transmediatic project for the achievement of university competences Jose L. Carreño Villada, Miguel Ángel Díaz Monsalvo

Los universitarios millennials: uso de redes sociales y relación con las marcas

University Millennials: use of social media and engage with brands

Pedro Pablo Marín Dueñas, Esther Simancas González

Ali Ferzat: De la caricatura comunicativa en papel a la caricatura activista en los medios digitales

Ali Ferzat: From political cartoons in papers to political cartoons in digital media

Salud Adelaida Flores Borjabad

Direito à informação e literacia midiática: Reflexões sobre a questão do acesso

Right to information and media literacy: Reflections on the question of acce

Christiane Delmondes Versuti 
Eficacia de los influencers como recurso publicitario en la estrategia de los anunciantes locales Efficacy of influencers as an advertising resource in the strategy of local advertisers

Isabel Iniesta-Alemán

As redes sociais como ferramenta de marketing em instituições de ensino superior no Brasil Social networks as a marketing tool in higher education institutions in Brazil

Alcino Ricoy JR, Rogério Eduardo Rodrigues Bazi

Estudio sobre la conceptualización y el tratamiento informativo de la violencia de género en la prensa digital cubana

Study about the conceptualization and information treatment of gender violence in the Cuban digital press

Regla Ismaray Cabreja Piedra, Karina Escalona Peña

\section{ARTÍCULOS ARTICLES}

Uso de Twitter durante los debates electorales televisados en los comicios andaluces de 2018 The use of Twitter during the televised electoral debates in the 2018 Andalusian elections Julia Fontenla Pedreira, Erika Conde Vázquez, Carmen Máiz Bar

Quem averigua as notícias, os algoritmos ou jornalistas? A lógica crítica de C. S.

Peirce como processo de identificação de uma Fake News

Who checks the news, algorithms or journalists? The critical logic of C. S. Peirce as a

process for identifying a Fake News

Adelino de Castro Oliveira Simões Gala, Vania Baldi, Universidad de Aveiro

\section{RESEÑAS REVIEWS}

Nuevo ecosistema comunicativo digital: El consumidor

Juan Carlos Figuereo Benítez

Corpus toponímic de Beniarrés

Bianca Sánchez-Gutiérrez

The Future Computed. La inteligencia artificial y su papel en la sociedad y Pulsa actualizar. La aventura de redescubrir el alma de Microsoft y concebir un futuro mejor para todos 


\title{
Uso de Twitter durante los debates electorales televisados en los comicios andaluces de 2018
}

\section{The use of Twitter during the televised electoral debates in the 2018 Andalusian elections}

Julia Fontenla Pedreira, Universidade de Vigo. Facultade de Ciencias Sociais e da Comunicación. Campus de Pontevedra A Xunqueira s/n.

$$
36005 \text { - Pontevedra }
$$

julia.fontenla.pedreira@uvigo.es | Orcid: https://orcid.org/0000-0001-8770-4761

\author{
Erika Conde Vázquez, Universidade de Vigo. Facultade de Ciencias Sociais e \\ da Comunicación. Campus de Pontevedra A Xunqueira s/n. \\ 36005 - Pontevedra \\ erikaconde@uvigo.es | Orcid: https://orcid.org/0000-0002-4898-3689 da Comunicación. Campus de Pontevedra A Xunqueira s/n.$$
36005 \text { - Pontevedra }
$$
maizbar@uvigo.es | Orcid: https://orcid.org/0000-0002-4357-4608 \\ Carmen Máiz Bar, Universidade de Vigo. Facultade de Ciencias Sociais e
}

DOI: http://dx.doi.org/10.12795/Ambitos.2019.i46.12

\section{Resumen}

Twitter está consolidado como medio de comunicación política, despertando interés de candidatos y partidos por su capacidad de movilizar a los ciudadanos y articular una campaña a favor de sus propios intereses (Kreiss, 2016). Numerosas investigaciones 
han analizado su poder como herramienta de comunicación y polarización política (Congosto-Martínez, 2016; Moya-Sánchez \& Herrera-Damas, 2016), así como herramienta de debate [estudios de contenido (Moe, 2012), estudios de sentimiento (Stieglitz\&DangXuan, 2013).

Este trabajo analizará cómo las fuerzas políticas PP, PSOE, Ciudadanos, Podemos y Vox han utilizado Twitter durante la retransmisión de los debates electorales de los comicios andaluces, celebrados el dos de diciembre de 2018. Se hará un seguimiento de los perfiles de los cuatro partidos que participaron en los debates retransmitidos por CanalSur y TVE los días 19 y 26 de noviembre, así como de Vox, la fuerza que irrumpe en el gobierno contra todo pronóstico. Se prestará atención a contenido y flujo de tuits de cada perfil, retuits, respuestas y hashtags, para comprobar si el uso de esta red social ha servido para predecir la tendencia política (Deltell et al, 2013). Además de los datos cuantitativos, se analizarán las estrategias seguidas por los partidos políticos en sus perfiles durante la emisión de los debates televisados.

Los resultados servirán para establecer un marco general de análisis de cómo esta red ha influido en la comunicación de los partidos en campaña, así como detectar el interés que suscitan entre su audiencia los temas que se plantean durante ambos debates.

\begin{abstract}
Twitter is consolidated as a political media, generating interest among candidates and parties due to its capability for mobilizing citizens and articulating a campaign in support of their own interests (Kreiss, 2016). Several studies have analyzed its power as a tool for communication and political polarization (Congosto-Martínez, 2016; Moya-Sánchez \& Herrera-Damas, 2016), as well as a tool for debate [content studies (Moe, 2012), sentiment studies (Stieglitz \& Dang-Xuan, 2013)].

This paper will analyze how the political parties PP, PSOE, Ciudadanos, Podemos and Vox have used Twitter during the broadcasting of the electoral debates for the AndaIusian elections, held December $2^{\text {nd }}, 2018$. A monitoring of the profiles of the four parties which participated in the debates televised by CanalSur and TVE on November $19^{\text {th }}$ and $26^{\text {th }}$ will be performed, as well as of that of Vox, given that, against all odds, it has entered the Government. Content, flow of tweets by each profile, retweets, answers and hashtags will be reviewed to find out whether the use of this social network could predict the political tendency (Deltell et al, 2013). In addition to the quantitative data, the strategies followed by the political parties in their profiles during the broadcasting of the electoral debates will be analyzed.

The results will be useful for establishing a general analysis framework on how this social network has influenced the communication of the parties during the campaign, as well as for detecting the interest that the different topics proposed during both debates raise among its audience.
\end{abstract}


Palabras clave: Twitter, redes sociales, elecciones Andalucía, debate electoral, comunicación política

Keywords: Twitter, social networks, Andalusian elections, electoral debate, political communication

\section{INTRODUCCIÓN Y MARCO TEÓRICO}

\subsection{Del discurso a la persuasión en red}

El factor persuasivo ha sido determinante en el discurso y lenguaje de los políticos a lo largo de la historia, obteniendo un alcance que estará supeditado a la capacidad de seducción de las palabras (Grijelmo, 2000) y que ejerce gran influencia en el estudio de la psicología, el comportamiento humano y sobre los sentimientos generados a consecuencia de las emociones. De acuerdo con la teoría de la inteligencia afectiva (Marcus, 2002), estas emociones se anticipan a las decisiones políticas y constituyen un requisito previo para el ejercicio de la razón.

Desde la década de los 60 se han analizado las relaciones entre lenguaje e ideología a través de distintos enfoques. Entre los más destacados cabe mencionar el de la escuela francesa de análisis crítico del discurso, centrada en la perspectiva de estudio del lenguaje político e influenciada por la lingüística estructural; no pasan desapercibidos otros como el de Lacan sobre el psicoanálisis, el materialismo histórico de Foucault, o la teoría de la enunciación y pragmática anglosajona. Por otro lado, la aportación de Chomsky ha sido una de las más revolucionarias, concibiendo el lenguaje como un fenómeno integrado dentro de las capacidades cognitivas humanas (Croft\&Cruse, 2008, p.17). Determinante también ha sido la hermenéutica, que analiza las posibles conexiones entre lenguaje, discurso y pensamiento, y propone la interpretación del lenguaje a tres niveles: lo que el lenguaje dice, lo que oculta y lo que revela o traiciona en sus significados dentro de un determinado contexto. Su propósito es descubrir la interconexión entre ideología y realidad, teniendo en cuenta que el discurso político no es solamente comunicación, sino también un medio de dominación y poder social, y que las estructuras lingüísticas constituyen un medio para la acción política (López Eire\&Guervós, 2000), convirtiendo así el arte de la retórica en un medio de persuasión.

En esta relación entre lenguaje, discurso y pensamiento surgen también perspectivas novedosas en el ámbito de las neurociencias aplicadas al estudio de la comunicación (neurocomunicación) y el lenguaje (neurolingüística). Los textos tradicionales incorporarán el uso de nuevas tecnologías, lenguajes y metalenguajes de la denominada sociedad en red (hipertextualidad) en un intento de caminar hacia el cerebro digital, que significa, en palabras de Small y Vorgan (2009), un desarrollo de este en función de las 
tecnologías de comunicación e información trasladadas a su vez a la conformación de los lenguajes.

La investigación en neurociencias ha facilitado la investigación experimental en marketing y comunicación gracias a la utilización de distintas técnicas y herramientas. Las técnicas biométricas, por ejemplo, han permitido observar qué ocurre en el cerebro de los consumidores y conocer de primera mano el comportamiento de los usuarios, e incluso gustos y sensaciones. En España, uno de los trabajos más destacados que emplea esta herramienta ha sido el análisis de los rasgos suicidas de la actriz Marilyn Monroe a partir de sus diarios (Rúas-Araújo et al., 2016); con posterioridad, se aplicó también para el análisis de discursos políticos o del estilo lingüístico de los candidatos del PP y el PSOE a la presidencia del Gobierno, comparando sus intervenciones en los debates televisados de las elecciones generales de 2011 y 2008 (Carrera et al, 2013).

En cualquier caso, si los nuevos medios de comunicación y las redes sociales pueden competir con los medios tradicionales en las campañas electorales sigue siendo una cuestión poco clara, aunque se reconoce su importancia en el diseño de las estrategias de campaña y la existencia de una convergencia e interacción dinámica, que facilita la intermediación de contenidos y la coexistencia de múltiples intermediarios en la relación entre las redes sociales y los medios de comunicación tradicionales (ChiaShin, 2016), dada su capacidad de combinación de texto e imágenes.

Redes sociales como Facebook se están convirtiendo en plataformas de politización que sirven para transmitir demandas personales y crear fuertes niveles de interacción (De la Fuente, 2010). El auge de la sociedad ha inspirado al desarrollo de la Democracia 2.0, compuesta por dos ejes: transversalidad y receptividad (Domínguez, 2009). Por ello, las redes sociales son hoy un elemento esencial en la estrategia de las campañas políticas, una oportunidad para la configuración de formas de interacción social gracias a intercambios dinámicos (Rizo, 2003).

Internet y redes sociales marcan el inicio de la etapa postmoderna de la comunicación política, ya que no sólo han introducido nuevas posibilidades de comunicación, sino que han transformado el estado puro de la política, la relación de política y medios (HoltzBacha, 2013), así como de los medios con las élites del poder (Mazaira et al, 2018), dentro de un proceso de hibridación y convergencia mediática entre nuevos medios y medios tradicionales, y de empoderamiento ciudadano (Casero-Ripollés, 2017).

El último Estudio Anual de Redes Sociales de IAB Spain (2018), muestra que el $85 \%$ de los internautas entre 16 y 65 años usa redes sociales, lo que equivale a un total de 25,5 millones de personas en España. El último Informe de Tendencias Digitales realizado por la misma entidad (IAB, 2019) señala que la denominada Generación Z (16 a 23 años), utiliza un mayor número de redes sociales a la vez y combina más dispositivos que la generación anterior, los Millenials (24 a 38 años), con una tendencia cada vez 
más acentuada al consumo de contenidos e información más rápida, menos permanente y hacia la denominada Social TV, a través de video y streaming.

De ahí la necesidad de contribuir al desarrollo de un modelo de medios de comunicación públicos y democráticos (Horowitz, 2015), dotándolos de recursos necesarios para innovar y renovarse, para lo cual es necesario contar con una buena participación del público (Tremblay, 2016), generando conversación con la audiencia que facilite emprendimiento, atención a la diversidad, independencia, universalidad y servicio al interés público a través de una política mediática activa (Trappel, 2016), fortaleciendo la relación entre emisor y receptor (Bruun, 2016) y que a su vez responda a las mutaciones tecnológico-sociales y a la formación de comunidades virtuales (Marzal\& Zallo, 2016).

\subsection{La argumentación seductora de Twitter}

Twitter es un medio ligado a la actualidad y uno de los mecanismos de comunicación más poderosos de la historia (Orihuela, 2011, p.15). Se trata de un canal de información frecuentemente utilizado por periodistas y políticos, aunque también empleado por estos últimos como medio en el que realizar declaraciones a través de sus cuentas personales. En Twitter prevalecen la espontaneidad y la inmediatez (rasgos clave en el fomento del intercambio fluido de conversación y debate político), pero esta red también ha convertido a las audiencias en actores participativos, activos y reivindicativos, en usuarios que "hablan en alto para que todo el mundo los oiga" (Congosto et al, 2011). Estamos ante internautas que hacen oír su voz, votantes que escuchan pero también hablan, e incluso en ocasiones, se convierten en influencers políticos, líderes de opinión en su entorno cercano.

En la actualidad el uso táctico y planificado de esta red es una realidad que ningún partido deja de lado, seduciéndolos a través de las posibilidades comunicativas que ofrece (Rodríguez \& Ureña, 2011). El entorno político no le da la espalda a este medio, a veces por convicción, otras por conveniencia: quiere ser escuchado para conseguir objetivos en clave electoral. En este ámbito político el querer ser escuchado sin barreras intermedias o dar su propio titular, prevalece por encima del escuchar o generar debate, consolidando la idea de que los políticos están más interesados en la difusión de su propia información y en conseguir retuits que en el propio debate (Campos-Domínguez, 2017), motivo por el que se le otorga importancia a los debates televisivos.

En período electoral Twitter se convierte en una herramienta mucho más incandescente, no sólo por el juego provocado entre partidos y candidatos, sino porque este altavoz mediático se considera el termómetro social predictor del comportamiento de la opinión pública. Zarella (2010) destacaba que los candidatos de la campaña electoral estadounidense con más seguidores en Twitter conseguían mejores resultados; en esta línea se posiciona Tumasjan (2010) quien determinó que en las elecciones alemanas de 2009 
los resultados obtenidos en las urnas estaban correlacionados con los candidatos o partidos más mencionados. En el caso español, la tendencia del uso de Twitter con fines electoralistas es más tardía, y el termómetro social ha girado alrededor de focos polarizados, creando campañas desorbitadas de insultos y aversiones. Las elecciones generales de abril de 2019 son un claro ejemplo, donde el odio social y la polémica se pusieron de manifiesto a través de usuarios defensores de partidos opuestos como VOX y Podemos, detectando a través de herramientas de minería de opinión el sentimiento positivo o negativo de cada tuit.

\section{OBJETIVOS Y METODOLOGÍA}

Esta investigación tiene como objetivo general (OG1) hacer un seguimiento cuantitativo y cualitativo de la presencia, audiencia, conversación y sentimiento en la red social Twitter de los partidos políticos andaluces (PP, PSOE, Ciudadanos, Adelante Andalucía y VOX), así como de sus respectivos candidatos (Juanma Moreno, Susana Díaz, Juan Marín, Teresa Rodríguez y Francisco Serrano) durante los debates televisados retransmitidos los días 19 y el 26 de noviembre de 2018 por CanalSur y TVE respectivamente.

Para ello, se han tenido en cuenta los tuits registrados en cada uno de los perfiles el día previo, durante y posterior al debate. Se ha desarrollado un volcado de contenido de un total de 1.750 tuits a través de la herramienta Twlets (893 asociados a las jornadas seleccionadas del primer debate, 857 al segundo), de los cuales se han seleccionado temáticamente aquellos de contenido exclusivamente relacionado con los debates de los comicios andaluces.

Se parte de la hipótesis de que los partidos políticos y sus candidatos emplean Twitter para conseguir la movilización de los votantes, y que esta red les sirve de plataforma de difusión de su ideología o como medio para tratar de desvalorizar a sus contrincantes. A partir de esta idea se establecen los siguientes objetivos específicos (OE):

- OE1: Analizar cuantitativamente la presencia, actividad y audiencia de los contenidos relacionados con los debates electorales realizados con motivo de los comicios andaluces, en las cuentas de Twitter de los partidos políticos seleccionados y sus candidatos, con el fin de establecer una dinámica de uso.

- OE2: Analizar cuáles son los tuits de mayor engagement (likes, comments y shares) de cada uno de los perfiles propuestos y, a partir de estos, realizar un análisis cualitativo en términos de conversación y sentimiento con el fin de conocer si existe interacción entre partidos/candidatos con los internautas.

- OE3: Analizar si el uso de Twitter de los perfiles propuestos es igual o si difiere en ambos debates, además de poder establecer diferencias entre partidos y candidatos. 
- OE4: Establecer cuáles son las estrategias de comunicación empleadas en los perfiles analizados (en este caso tan solo del propio partido) durante la retransmisión de los dos debates electorales entre candidatos.

Para conocer la repercusión de los tuits políticos es necesario atender al engagement (índice de respuesta de los usuarios sobre las publicaciones realizadas) de las publicaciones. González (2013, p.51) señala que "las interacciones en la red social no son únicamente las veces que se comparte el contenido en el perfil de un usuario sino también la cantidad de veces que esos contenidos se comentan, ganan un 'me gusta' o lo comparten otras personas". Por su parte Valerio et al. (2015), indican que una participación más activa se consigue compartiendo mensajes, ya que se fomenta la difusión, pero también otorga significado y afinidad hacia ese mensaje por parte del internauta. Analizamos el nivel de engagement mediante la fórmula de total de interacciones/total de seguidores $\times 100$.

Además, se atenderá al sentimiento (sentiment) generado en las publicaciones. Este sentimiento con carga positiva, negativa o neutra se realiza a través de sistemas big data que atienden las publicaciones y son capaces de entender interpretar, discernir y traducir el significado de un texto digital, haciendo posible su análisis, así como clasificarlo en objetivo o subjetivo; y en la categoría de los subjetivos, en positivos o negativos. El resultado obtenido servirá para clasificarlos dentro de estas categorías, así, la polaridad (el sentimiento en sí) se establece entre valores de -1 a +1 . La subjetividad antes mencionada consiste en una medida del sentimiento siendo objetivo a subjetivo, entre valores de 0 a 1. Hay que tener en cuenta que los algoritmos empleados no entienden de ironía, humor o sarcasmo, por lo que no es difícil encontrar falsos positivos o falsos negativos (Sobrino-Sande, 2018).

Por otro lado, se establecen medias entre los valores encontrados, compound, por lo que un sentimiento positivo correspondería a un compound $>=0.5$; sentimiento neutro compound $>-0.5$ y sentimiento negativo $a<0.5$.

\section{RESULTADOS}

Los 10 perfiles analizados arrojan en el período propuesto (día previo, durante y día después de cada debate) un total de 1.750 tuits, en los que los hashtags más utilizados están relacionados con el propio debate del canal (\#DebateCanalSur, \#DebateAndaluciaRTVE). 
Tabla 1. Muestra perfiles partidos

\begin{tabular}{|c|c|c|c|}
\hline & Seguidores & Tuits & $\begin{array}{c}\text { Hashtags más emplea- } \\
\text { dos }\end{array}$ \\
\hline PP Andaluz @ppandaluz & 37.000 & 275 & $\begin{array}{c}\text { \#debatecanalsur \#2D } \\
\text { \#VotaGarantiadeCambio } \\
\text { \#DebateAndaluciaRTVE }\end{array}$ \\
\hline $\begin{array}{l}\text { PSOE @psoedeandalu- } \\
\text { cia }\end{array}$ & 46.700 & 524 & $\begin{array}{c}\text { \#DebateCanalSur } \\
\text { \#VamosConSusana } \\
\text { \#SusanaPorAndalucía } \\
\text { \#VotaPSOE } \\
\text { \#DebateAndaluciaRTVE }\end{array}$ \\
\hline $\begin{array}{l}\text { Ciudadanos } \\
@ \text { Cs_Andalucia }\end{array}$ & 25.500 & 382 & $\begin{array}{c}\text { \#debatecanalsur \#2D } \\
\text { \#Andalucía } \\
\text { \#DebateAndaluciaRTVE }\end{array}$ \\
\hline $\begin{array}{l}\text { Adelante Andalucía } \\
\text { @AdelanteAND }\end{array}$ & 12.000 & 188 & $\begin{array}{c}\text { \#EleccionesAndalucia2D } \\
\text { \#AdelanteAndalucia } \\
\text { \#DebateAndaluciaRTVE } \\
\text { \#DebateCanalSur } \\
\text { \#2DCanalSur }\end{array}$ \\
\hline VOX \#AndaluciaVox & 3.517 & \multicolumn{2}{|c|}{$\begin{array}{c}\text { Cuenta registrada a inicios de } 2019 \text {, no existen } \\
\text { publicaciones relativas a los comicios }\end{array}$} \\
\hline
\end{tabular}

Elaboración propia a partir de datos de Twitter

Tabla 2. Muestra perfiles candidatos

\begin{tabular}{|l|c|c|c|}
\hline & Seguidores & Tuits & $\begin{array}{c}\text { Hashtags más emplea- } \\
\text { dos }\end{array}$ \\
\hline $\begin{array}{l}\text { Juanma Moreno } \\
\text { @JuanMa_Moreno } \\
\text { (PP) }\end{array}$ & 10.400 & 113 & $\begin{array}{c}\text { \#debatecanalsur } \\
\text { \#VotaGarantíadeCambio } \\
\text { \#DebateAndaluciaRTVE }\end{array}$ \\
\hline $\begin{array}{l}\text { Susana Díaz } \\
\text { @usanadiaz (PSOE) }\end{array}$ & 175.000 & 11 & $\begin{array}{c}\text { \#SusanaPorAndalucía } \\
\text { \#VotaPSOE }\end{array}$ \\
\hline
\end{tabular}


Tabla 2. (Continuación)

\begin{tabular}{|l|c|c|c|}
\hline $\begin{array}{l}\text { Juan Marín @JuanMa- } \\
\text { rin_Cs } \\
\text { (Ciudadanos) }\end{array}$ & 19.900 & 31 & $\begin{array}{c}\text { AhoraSiCs } \\
\text { \#DebateCanalSur } \\
\text { \#bebateAndaluciaRTVE }\end{array}$ \\
\hline $\begin{array}{l}\text { Teresa Rodríguez } \\
\text { @TeresaRodr_ } \\
\text { (Adelante Andalucía) }\end{array}$ & 317.000 & 216 & $\begin{array}{c}\text { \#AdelanteAndalucía } \\
\text { \#TeresaPresidenta } \\
\text { \#DebateCanalSur } \\
\text { \#DebateAndaluciaRTVE }\end{array}$ \\
\hline $\begin{array}{l}\text { Francisco Serrano } \\
\text { FcoSer1 (VOX) }\end{array}$ & 944 & \multicolumn{2}{|c|}{ Cuenta sin uso desde 2015 } \\
\hline
\end{tabular}

Elaboración propia a partir de datos de Twitter

Por agrupación y candidato se recogieron los siguientes resultados:

\subsection{PP Andaluzl @ppandaluz}

El perfil del PP andaluz ha registrado un total de 128 tuits durante las jornadas previa, post y durante el primer debate en CanalSur (todos ellos con contenido relativo al propio debate o campaña), donde la mayor actividad se centra en igual medida en el día anterior y en el mismo día de celebración del debate (19 de noviembre), con un $70 \%$ de contenido electoral. En el caso del segundo debate, en RTVE, el número de tuits es similar (147) y con mismo porcentaje de contenido electoral (70\%).

Tras hacer el volcado de tuits, se comprueba que los que tienen mayor engagement pertenecen al primer debate o a su jornada posterior en la misma medida. Los temas destacados giran en torno a propuestas de Susana Díaz durante el debate y reacciones a su persona. En estos tuits se registra el sentimiento nivel más alto en 0.28 puntos, siendo el sentiment con valor negativo predominante.

Tabla 3. Tuits primer debate perfil @ppandaluz

\begin{tabular}{|l|c|c|c|}
\hline & $\begin{array}{c}\text { Jornada previa } \\
\mathbf{1 8 / 1 1 / 2 0 1 8}\end{array}$ & $\begin{array}{c}\text { Jornada debate } \\
\mathbf{1 9 / 1 1 / 2 0 1 9}\end{array}$ & $\begin{array}{c}\text { Jornada posterior } \\
\mathbf{2 0 / 1 1 / 2 0 1 8}\end{array}$ \\
\hline Tuits & 52 & 52 & 24 \\
\hline $\begin{array}{l}\text { Tuits con contenido debate o } \\
\text { campaña }\end{array}$ & 41 & \multicolumn{2}{|c|}{ Todos } \\
\hline
\end{tabular}

Elaboración propia a partir de datos de Twitter 
Tabla 4. Tuits segundo debate perfil @ppandaluz

\begin{tabular}{|l|c|c|c|}
\hline & $\begin{array}{c}\text { Jornada previa } \\
\mathbf{2 5 / 1 1 / 2 0 1 8}\end{array}$ & $\begin{array}{c}\text { Jornada debate } \\
\mathbf{2 6 / 1 1 / 2 0 1 9}\end{array}$ & $\begin{array}{c}\text { Jornada posterior } \\
\mathbf{2 7 / 1 1 / 2 0 1 8}\end{array}$ \\
\hline Tuits & 14 & 77 & 56 \\
\hline $\begin{array}{l}\text { Tuits con contenido de- } \\
\text { bate o campaña }\end{array}$ & \multicolumn{2}{|c|}{ Todos } \\
\hline
\end{tabular}

Elaboración propia a partir de datos de Twitter

Tabla 5. Tuits con mayor engagement en perfil @ppandaluz y sentiment generado

\begin{tabular}{|l|c|c|c|c|c|}
\hline & Engage & $\begin{array}{c}\text { Com- } \\
\text { pound }\end{array}$ & $\begin{array}{c}\text { Nega- } \\
\text { tivo }\end{array}$ & $\begin{array}{c}\text { Neu- } \\
\text { tro }\end{array}$ & $\begin{array}{c}\text { Posi- } \\
\text { tivo }\end{array}$ \\
\hline $\begin{array}{l}\text { 19/11/2018- Les quito la acreditación y se } \\
\text { van. ¡Devuélvanme la acreditación! } \\
\begin{array}{l}\text { Cuando en Andalucía hablábamos del "regi- } \\
\text { men del PSOE Andaluz" no es por gusto. } \\
\text { \#Cortijadas }\end{array}\end{array}$ & 0,287 & $-0,359$ & 0,098 & 0,902 & 0 \\
\hline $\begin{array}{l}\text { 20/11/2018- Casi nadie se dio cuenta ano- } \\
\text { che en el debate de CanalSur, pero hubo un } \\
\text { momento en el que las cámaras pillaron a } \\
\text { Susana Díaz diciéndole a Juan Marín que } \\
\text { contestara él a lo que estaba diciendo } \\
\text { Juanma Moreno. EL "ordeno y mando" si- } \\
\text { gue activo. }\end{array}$ & 0,180 & 0 & 0 & 0 & 0 \\
\hline $\begin{array}{l}\text { 19/11/2018- \#DebateCanalSur ¿Por qué } \\
\text { Susana Díaz no responde a las preguntas } \\
\text { de @JuanMa_Moreno? } \\
\text { Con sus silencios se convierte en cómplice. }\end{array}$ & 0,145 & $-0,296$ & 0,104 & 0,896 & 0 \\
\hline $\begin{array}{l}\text { 19/11/2018- \#DebateCanalSur. Ciudadanos } \\
\text { ha tirdo a la basura la oportunidad de con- } \\
\text { trolar y fiscalizar a la Sra. Díaz durante esta } \\
\text { última legislature. @JuanMa_Moreno }\end{array}$ & 0,136 & 0,34 & 0 & 0,897 & 0,103 \\
\hline
\end{tabular}

Elaboración propia a partir de datos de Twitter 


\subsection{Juanma Moreno/@JuanMa_Moreno}

El perfil del candidato por el PP de Andalucía, Juanma Moreno, registra un total de 52 tuits durante las jornadas previa, post y durante el debate de CanalSur (todos ellos con contenido relativo al propio debate o campaña) donde la mayor actividad se centra durante el día de celebración del debate, 19 de noviembre. Durante el debate de RTVE, el número de tuits es similar (61) con $100 \%$ de contenido electoral, donde prevalecen los generados durante la celebración del debate -en esta fase por personas a cargo de la gestión del perfil, pues Juanma Moreno estaba en pantalla.

Los tuits con mayor engagement pertenecen al primer debate, destacando el tema "PP garantía de cambio". En estos tuits se registra el mayor nivel de engagement en 1,19 puntos, siendo el sentiment con valor cero predominante.

Tabla 6. Tuits segundo debate perfil @JuanMa_Moreno

\begin{tabular}{|l|c|c|c|}
\hline & $\begin{array}{c}\text { Jornada previa } \\
\mathbf{2 5 / 1 1 / 2 0 1 8}\end{array}$ & $\begin{array}{c}\text { Jornada debate } \\
\mathbf{2 6 / 1 1 / 2 0 1 9}\end{array}$ & $\begin{array}{c}\text { Jornada posterior } \\
\mathbf{2 7 / 1 1 / 2 0 1 8}\end{array}$ \\
\hline Tuits & 10 & 31 & 1 \\
\hline $\begin{array}{l}\text { Tuits con contenido de- } \\
\text { bate o campaña }\end{array}$ & \multicolumn{3}{|c|}{ Todos } \\
\hline
\end{tabular}

Elaboración propia a partir de datos de Twitter

Tabla 7. Tuits con mayor engagement en perfil @JuanMa_Moreno y sentiment generado

\begin{tabular}{|l|c|c|c|c|c|}
\hline & Engage & $\begin{array}{c}\text { Com- } \\
\text { pound }\end{array}$ & Negativo & Neutro & Positivo \\
\hline $\begin{array}{l}19 / 11 / 2018 \text { - Soy Juanma Moreno y esta es mi } \\
\text { historia. \#VotaGarantíaDeCambio }\end{array}$ & 1,196 & 0 & 0 & 1 & 0 \\
\hline $\begin{array}{l}19 / 11 / 2018-\text { ¿Susana Díaz cree que España } \\
\text { es un Estado plurinacional? Los libros de } \\
\text { texto de los niños andaluces dicen que "Es- } \\
\text { paña es un Estado plurinacional" \#DebateCa- } \\
\text { nalSur }\end{array}$ & 0,823 & 0 & 0 & 1 & 0 \\
\hline
\end{tabular}


Tabla 7. (Continuación)

\begin{tabular}{|l|c|c|c|c|c|}
\hline $\begin{array}{l}\text { 19/11/2018- Buenas noches Andalucía ¿Con- } \\
\text { sideran que el PSOE debe continuar otros 4 } \\
\text { años más en el Gobierno de la Junta o es mo- } \\
\text { mento de cambiar? Siquieres cambiar, la } \\
\text { ñunica \#GarantíaDeCambio es el @PPanda- } \\
\text { luz }\end{array}$ & 0,79 & 0 & 0 & 1 & 0 \\
\hline $\begin{array}{l}\text { 20/11/2018- Me dijo la señora Díaz que no } \\
\text { era cierto, pero esta es la prueba de lo que se } \\
\text { enseña a los niños andaluces. Lamentable. }\end{array}$ & 0,1768 & $-0,571$ & 0,176 & 0,824 & 0 \\
\hline $\begin{array}{l}\text { 26/11/2018- Esta es la tasa de empleo desde } \\
\text { 1996. Cuando el Partido Popular gobierna en } \\
\text { España, el paro ha bajado en Andalucía. \#Vo- } \\
\text { taGarantíaDeCambio \#DebateAndaluciaRTVE }\end{array}$ & 0,668 & 0,636 & 0 & 0,809 & 0,191 \\
\hline
\end{tabular}

Elaboración propia a partir de datos de Twitter

\subsection{PSOE/ @psoedeandalucia}

En el caso del PSOE, se han registrado un total de 273 tuits durante las jornadas previa, post y durante el debate de CanalSur (todos ellos con contenido relativo al propio debate o campaña), donde la mayor actividad se centra en el día de celebración, 19 de noviembre.

Durante el debate de RTVE, el número de tuits es similar (251), todos referidos a la temática del debate, excepto nueve que se centran en contenidos contra la violencia de género. Los tuits con mayor engagement pertenecen al segundo debate, con temas en torno a las propuestas de Susana Díaz y las reacciones a estas.

Tabla 8. Tuits primer debate perfil @psoedeandalucia

\begin{tabular}{|l|c|c|c|}
\hline & $\begin{array}{c}\text { Jornada previa } \\
\mathbf{1 8 / 1 1 / 2 0 1 8}\end{array}$ & $\begin{array}{c}\text { Jornada debate } \\
\mathbf{1 9 / 1 1 / 2 0 1 9}\end{array}$ & $\begin{array}{c}\text { Jornada posterior } \\
\mathbf{2 0 / 1 1 / 2 0 1 8}\end{array}$ \\
\hline Tuits & 68 & 146 & 59 \\
\hline $\begin{array}{l}\text { Tuits con contenido de- } \\
\text { bate o campaña }\end{array}$ & \multicolumn{3}{|c|}{ Todos } \\
\hline
\end{tabular}

Elaboración propia a partir de datos de Twitter 
Tabla 9. Tuits segundo debate perfil @psoedeandalucia

\begin{tabular}{|l|c|c|c|}
\hline & $\begin{array}{c}\text { Jornada previa } \\
\mathbf{2 5 / 1 1 / 2 0 1 8}\end{array}$ & $\begin{array}{c}\text { Jornada debate } \\
\mathbf{2 6 / 1 1 / 2 0 1 9}\end{array}$ & $\begin{array}{c}\text { Jornada posterior } \\
\mathbf{2 7 / 1 1 / 2 0 1 8}\end{array}$ \\
\hline Tuits & 17 & 131 & 103 \\
\hline $\begin{array}{l}\text { Tuits con contenido de- } \\
\text { bate o campaña }\end{array}$ & 9 & \multicolumn{2}{|c|}{ Todos } \\
\hline
\end{tabular}

Elaboración propia a partir de datos de Twitter

Tabla 10. Tuits con mayor engagement en perfil @psoeandalucia y sentiment generado

\begin{tabular}{|c|c|c|c|c|c|}
\hline & $\begin{array}{c}\text { En- } \\
\text { gage }\end{array}$ & $\begin{array}{l}\text { Com- } \\
\text { pound }\end{array}$ & $\begin{array}{c}\text { Nega- } \\
\text { tivo }\end{array}$ & $\begin{array}{c}\text { Neu- } \\
\text { tro }\end{array}$ & $\begin{array}{c}\text { Posi- } \\
\text { tivo }\end{array}$ \\
\hline $\begin{array}{l}\text { 26/11/2018- MINUTO FINAL @susanadiaz en el } \\
\text { \#DebateAndaluciaRTVE. Queremos \#MásAnda- } \\
\text { lucia donde los \#andaluces y \#andaluzas vivan } \\
\text { major. Sin bloqueos y con estabilidad. Por un } \\
\text { Gobierno sólido y solvent } \\
\text { DALE AL PLAY y \#VotaPSOE \#2D \#SusanaPo- } \\
\text { rAndalucia }\end{array}$ & 1,586 & $-0,119$ & 0,098 & 0,817 & 0,085 \\
\hline $\begin{array}{l}\text { 26/11/2018- Donde la \#derecha gobierna. XRe- } \\
\text { cortes y privatización. XLos libros de texto no } \\
\text { son gratis y no se bonifican matrículas universita- } \\
\text { rias } \\
\text { XNo se aportan fondos a Ley de \#-dependencia } \\
\text { (Y mucho más) @susanadiaz lo cuenta \#Deba- } \\
\text { teAndaluciaRTVE \#MasAndalucia }\end{array}$ & $\begin{array}{c}01,04 \\
0\end{array}$ & $-0,659$ & 0,162 & 0,809 & 0,029 \\
\hline $\begin{array}{l}\text { 27/11/2018- Nadie duda de quién va a ganar en } \\
\text { Andalucúa pero, si queremos que no haya blo- } \\
\text { queo, temenos que ganar bien. Hay que recoger } \\
\text { la papeleta y votar al @PSOE y a @susanadiaz. } \\
\text { Me siento orgulloso de la acción socialista en la } \\
\text { Junta. ¡A ganaren el \#2D y a gobernar! \#MásAn- } \\
\text { dalucía }\end{array}$ & $0,985 \mid$ & $-0,359$ & 0,054 & 0,946 & 0 \\
\hline
\end{tabular}


Tabla 10. (Continuación)

\begin{tabular}{|l|c|c|c|c|c|}
\hline $\begin{array}{l}\text { 27/11/2018- Acto de Susana Díaz en Marbe- } \\
\text { Ila \#MásAndalucía }\end{array}$ & 0,965 & 0 & 0 & 1 & 0 \\
\hline $\begin{array}{l}\text { 26/11/2018- Salgo satisfecha de un debate } \\
\text { en el que he planteado propuestas y respues- } \\
\text { tas para el future de \#Andalucía. PP y Ciuda- } \\
\text { danos no han querido contestarme, hasta en } \\
\text { cuatro ocasiones, si se apoyarian en la ultra- } \\
\text { derecha de VOX para bloquear un gobierno } \\
\text { no socialista. }\end{array}$ & 0,937 & $-0,296$ & 0,052 & 0,948 & 0 \\
\hline
\end{tabular}

Elaboración propia a partir de datos de Twitter

\subsection{Susana Díazl @susanadiaz}

El perfil de la candidata socialista registra un número muy bajo de tuits durante las jornadas previa, post y durante el debate de CanalSur, con 11 publicaciones $(65 \%$ de contenido electoral). La mayor actividad ocurre el día después. Durante el segundo debate el número de tuits es prácticamente igual (10), de los cuales $70 \%$ se centran en las reacciones de la candidata sobre el debate.

Tabla 11. Tuits primer debate perfil @susanadiaz

\begin{tabular}{|l|c|c|c|}
\hline & $\begin{array}{c}\text { Jornada previa } \\
\mathbf{1 8 / 1 1 / 2 0 1 8}\end{array}$ & $\begin{array}{c}\text { Jornada debate } \\
\mathbf{1 9 / 1 1 / 2 0 1 9}\end{array}$ & $\begin{array}{c}\text { Jornada posterior } \\
\mathbf{2 0 / 1 1 / 2 0 1 8}\end{array}$ \\
\hline Tuits & 1 & 3 & 7 \\
\hline $\begin{array}{l}\text { Tuits con contenido de- } \\
\text { bate o campaña }\end{array}$ & Todos & 2 & 5 \\
\hline
\end{tabular}

Elaboración propia a partir de datos de Twitter

Teniendo en cuenta los tuits más destacados según su engagement, se sitúa su nivel más alto en 0.89 puntos. No prevalecen tuits de ninguno de los dos debates, sino que se aprecia variedad en cuanto a ambos y girando en torno a la visión de la propia candidata. 
Tabla 12. Tuits segundo debate perfil @susanadiaz

\begin{tabular}{|l|c|c|c|}
\hline & $\begin{array}{c}\text { Jornada previa } \\
\mathbf{2 5 / 1 1 / 2 0 1 8}\end{array}$ & $\begin{array}{c}\text { Jornada debate } \\
\mathbf{2 6 / 1 1 / 2 0 1 9}\end{array}$ & $\begin{array}{c}\text { Jornada posterior } \\
\mathbf{2 7 / 1 1 / 2 0 1 8}\end{array}$ \\
\hline Tuits & 1 & 3 & 6 \\
\hline $\begin{array}{l}\text { Tuits con contenido de- } \\
\text { bate o campaña }\end{array}$ & 0 & 2 & 4 \\
\hline
\end{tabular}

Elaboración propia a partir de datos de Twitter

En los tuits con mayor engagement predomina el sentiment con valor 0 , lo que no posiciona a los usuarios que participan hacia el apoyo u oposición a esta candidata.

Tabla 13. Tuits con mayor engagement en perfil @susanadiaz y sentiment generado

\begin{tabular}{|c|c|c|c|c|c|}
\hline & Engage & $\begin{array}{l}\text { Com- } \\
\text { pound }\end{array}$ & $\begin{array}{c}\text { Nega- } \\
\text { tivo }\end{array}$ & $\begin{array}{c}\text { Neu- } \\
\text { tro }\end{array}$ & $\begin{array}{c}\text { Posi- } \\
\text { tivo }\end{array}$ \\
\hline $\begin{array}{l}\text { 18/11/2018- Gracias @sanchezcastejon por } \\
\text { venir a } \\
\text { \#Chiclana a apoyar nuestra campana en po- } \\
\text { sitive, con propuestas y a hablar de \#Andalu- } \\
\text { cía.Y un } \\
\text { abrazo a I@s compañeros de \#Cádiz por } \\
\text { aportarnos tanto calor y energía, que nos he- } \\
\text { mos pasado, desbordando el acto. Vuestro } \\
\text { apoyo nos motiva aún más. }\end{array}$ & 0,891 & 0 & 0 & 1 & 0 \\
\hline $\begin{array}{l}\text { 27/11/2018- Gracias a sanchezcastejon por } \\
\text { volver } \\
\text { a acompañarnos a I@s socialistas del } \\
@ \text { @soeandalucia en este gran mitin de \#Mar- } \\
\text { bella. } \\
\text { Salimos reforzad@s para ganar y bien las } \\
\text { elecciones el 2D. Lo haremos por nuestra tie- } \\
\text { rra, } \\
\text { para que nadie frene el avance y el future de } \\
\text { \#Andalucía. }\end{array}$ & 0,564 & 0 & 0 & 1 & 0 \\
\hline $\begin{array}{l}\text { 19/11/2018- Con mi equipo tras el debate en } \\
\text { @canalsur. Gracias a la television pública de } \\
\text { \#Andalucíapor permitrinos hablar de los } \\
\text { desafíos } \\
\text { y retos de nuestra tierra. }\end{array}$ & 0,454 & 0 & 0 & 1 & 0 \\
\hline
\end{tabular}




\section{Tabla 13. (Continuación)}

\begin{tabular}{|c|c|c|c|c|c|}
\hline $\begin{array}{l}\text { 25/11/2018- La violencia hacia las \#mujeres } \\
\text { es el vivo ejemplo de la desigualdad. Hoy, } \\
\text { |@s } \\
\text { socialistas ponemos voz y rostro a la lucha } \\
\text { que } \\
\text { mantenemos todo el año contra el machismo, } \\
\text { que } \\
\text { ha sido el terrorismo que más víctimas se ha } \\
\text { cobrado en este país. }\end{array}$ & & & & & \\
\hline \#DíaContraLaViolenciaDeGénero & 0,338 & 0,585 & 0 & 0,897 & 0,103 \\
\hline
\end{tabular}

Elaboración propia a partir de datos de Twitter

\subsection{Ciudadanos/ @Cs_Andalucia}

Ciudadanos Andalucía registra un total de 185 tuits durante las jornadas previa, post y durante el debate de CanalSur (todos ellos con contenido relativo al propio debate o campaña), donde la mayor actividad se centra el día posterior (20/11/2018). Durante el debate de RTVE, el número de tuits es similar (197), con $65 \%$ relativos al contenido del propio debate.

En cuanto a los tuits con mayor engagement, no prevalecen publicaciones de ninguno de los dos debates, sino que se aprecia un número similar entre ambos y, como tema principal, la "política útil" de Ciudadanos. En estos tuits se registra el nivel de engagement más alto en 0,69 puntos, teniendo el sentiment valor negativo.

Tabla 14. Tuits primer debate perfil @Cs_Andalucia

\begin{tabular}{|l|c|c|c|}
\hline & $\begin{array}{c}\text { Jornada previa } \\
\mathbf{1 8 / 1 1 / 2 0 1 8}\end{array}$ & $\begin{array}{c}\text { Jornada debate } \\
\mathbf{1 9 / 1 1 / 2 0 1 9}\end{array}$ & $\begin{array}{c}\text { Jornada posterior } \\
\mathbf{2 0 / 1 1 / 2 0 1 8}\end{array}$ \\
\hline Tuits & 60 & 43 & 82 \\
\hline $\begin{array}{l}\text { Tuits con contenido de- } \\
\text { bate o campaña }\end{array}$ & \multicolumn{3}{|c|}{ Todos } \\
\hline
\end{tabular}

Elaboración propia a partir de datos de Twitter 
Tabla 15. Tuits segundo debate perfil @Cs_Andalucia

\begin{tabular}{|l|c|c|c|}
\hline & $\begin{array}{c}\text { Jornada previa } \\
\mathbf{2 5 / 1 1 / 2 0 1 8}\end{array}$ & $\begin{array}{c}\text { Jornada debate } \\
\mathbf{2 6 / 1 1 / 2 0 1 9}\end{array}$ & $\begin{array}{c}\text { Jornada posterior } \\
\mathbf{2 7 / 1 1 / 2 0 1 8}\end{array}$ \\
\hline Tuits & 75 & 52 & 70 \\
\hline $\begin{array}{l}\text { Tuits con contenido de- } \\
\text { bate o campaña }\end{array}$ & 68 & \multicolumn{2}{|c|}{ Todos } \\
\hline
\end{tabular}

Elaboración propia a partir de datos de Twitter

Tabla 16. Tuits con mayor engagement en perfil @Cs_Andalucia y sentiment generado

\begin{tabular}{|l|c|c|c|c|c|}
\hline & $\begin{array}{c}\text { En- } \\
\text { gage }\end{array}$ & $\begin{array}{c}\text { Com- } \\
\text { pound }\end{array}$ & $\begin{array}{c}\text { Nega- } \\
\text { tivo }\end{array}$ & $\begin{array}{c}\text { Neu- } \\
\text { tro }\end{array}$ & $\begin{array}{c}\text { Posi- } \\
\text { tivo }\end{array}$ \\
\hline $\begin{array}{l}\text { 20/11/2018- @JuanMarin_Cs "Maria es una } \\
\text { teniente de la Guardia Civil, me dio esta pul- } \\
\text { sera en una manifestación de @JUSAPOL. } \\
\text { Le dije que no me la quitaría hasta que la } \\
\text { \#Equiparación fuera una realidad. Estoy } \\
\text { convencido de que me la quitaré cuando } \\
\begin{array}{l}\text { C's gobiernes España" @NewEcono- } \\
\text { myForum }\end{array}\end{array}$ & 0,692 & $-0,296$ & 0,049 & 0,951 & 0 \\
\hline $\begin{array}{l}\text { 20/11/2018- "Un gobierno sin prsupuestos } \\
\text { no gobierna nada”. "Gobernar no consiste } \\
\text { en vivir en La Moncloa". Miren a Sánchez } \\
\text { en un ataque de lucidez... Cuando no es- } \\
\text { taba ocupando La Moncloa, claro. Aplí- } \\
\text { quese el cuento: \#EleccionesYa }\end{array}$ & 0,650 & $-0,848$ & 0,254 & 0,746 & 0 \\
\hline $\begin{array}{l}\text { 18/11/2018- Gracias a @CiudadanosCs y } \\
\text { @Albert_Rivera por el apoyo a la @lL- } \\
\text { PJusapol. Hicieron suyo nuestro argumen- } \\
\text { tario "Mismo trabajo mismo salario". Xq es } \\
\text { justiciar, xq es una cuestión de dignidad y } \\
\text { de igualdad. 550.000 españoles nos apoya- } \\
\text { ron con su firma. \#EquiparacionYa }\end{array}$ & 0,60 & 0 & 00,254 & 0,746 & 0 \\
\hline
\end{tabular}


Tabla 16. (Continuación)

\begin{tabular}{|l|l|l|l|l|l|}
\hline $\begin{array}{l}\text { 26/11/2018- ¿Cansado de perder siempre? } \\
\text { Tras cuatro décadas de derrota, ha llegado } \\
\text { la hora de ganar. Ahora sí, gana con Ciuda- } \\
\text { danos }\end{array}$ & 0,575 & 0,34 & 0 & 0,902 & 0,098 \\
\hline $\begin{array}{l}\text { 19/11/2018- @JuanMarin_Cs "Yo no vengo } \\
\text { aquí a decir a los andaluces que le vamos a } \\
\text { bajar los impuestos, sino que ya los esta- } \\
\text { mos bajado. Desde la oposición hemos con- } \\
\text { seguido eliminar el imp. de sucesiones y ba- } \\
\text { jado el IRPF a los trabajadores" \#Debate- } \\
\text { CanalSurCiudadanos es \#PolíticaÚtil }\end{array}$ & 0,9498 & $-0,296$ & 0,053 & 0,947 & 0 \\
\hline
\end{tabular}

Elaboración propia a partir de datos de Twitter

\subsection{Juan Marín/ @JuanMarin_Cs}

El perfil del candidato de Ciudadanos, Juan Marín, registra un número bajo de tuits (19) durante las jornadas previa, post y durante el debate de CanalSur (todos ellos con contenido relativo al propio debate o campaña) y con actividad similar durante los tres días. Durante el debate de RTVE, el número de tuits es similar (12), con mayor actividad durante el día de celebración, con $90 \%$ relativos al contenido del debate.

Teniendo en cuenta las publicaciones con mayor engagement, sitúa su nivel más alto en 20 puntos (algo que se debe a que el número de seguidores es más bajo que en los demás perfiles $\mathrm{y}$, por tanto, el cálculo del engagement aumenta correlativamente). No prevalecen tuits de ninguno de los dos debates, sino que se aprecia variedad. Temáticamente, el contenido principal gira en torno el partido C's contra la corrupción y por el cambio de Andalucía. En estos tuits predomina el sentiment con valor negativo de los usuarios.

Tabla 17. Tuits primer debate perfil @JuanMarin_Cs

\begin{tabular}{|l|c|c|c|}
\hline & $\begin{array}{c}\text { Jornada previa } \\
\mathbf{1 8 / 1 1 / 2 0 1 8}\end{array}$ & $\begin{array}{c}\text { Jornada debate } \\
\mathbf{1 9 / 1 1 / 2 0 1 9}\end{array}$ & $\begin{array}{c}\text { Jornada posterior } \\
\mathbf{2 0 / 1 1 / 2 0 1 8}\end{array}$ \\
\hline Tuits & 6 & 5 & 8 \\
\hline $\begin{array}{l}\text { Tuits con contenido de- } \\
\text { bate o campaña }\end{array}$ & \multicolumn{3}{|c|}{ Todos } \\
\hline
\end{tabular}

Elaboración propia a partir de datos de Twitter 
Tabla 18. Tuits segundo debate perfil @JuanMarin_Cs

\begin{tabular}{|l|c|c|c|}
\hline & $\begin{array}{c}\text { Jornada previa } \\
\mathbf{2 5 / 1 1 / 2 0 1 8}\end{array}$ & $\begin{array}{c}\text { Jornada debate } \\
\mathbf{2 6 / 1 1 / 2 0 1 9}\end{array}$ & $\begin{array}{c}\text { Jornada posterior } \\
\mathbf{2 7 / 1 1 / 2 0 1 8}\end{array}$ \\
\hline Tuits & 3 & 6 & 3 \\
\hline $\begin{array}{l}\text { Tuits con contenido de- } \\
\text { bate o campaña }\end{array}$ & 2 & 5 & 3 \\
\hline
\end{tabular}

Elaboración propia a partir de datos de Twitter

Tabla 19. Tuits con mayor engagement en perfil @JuanMarin_Cs y sentiment generado

\begin{tabular}{|l|c|c|c|c|c|}
\hline & $\begin{array}{c}\text { En- } \\
\text { gage }\end{array}$ & Compound & Negativo & Neutro & $\begin{array}{c}\text { Posi- } \\
\text { tivo }\end{array}$ \\
\hline $\begin{array}{l}\text { 20/11/2018- Hace diez meses me rega- } \\
\text { laron una pulsera @juasapol que me } \\
\text { acompaña desde entonces. En ella, un } \\
\text { lema: "Prohibidorendirse". Lo prometí } \\
\text { por entonces, y lo reitero: no me la qui- } \\
\text { taré hasta que no se consiga la \#Equipa- } \\
\text { raciónYa para nuestras FFCC de Seguri- } \\
\text { dad. Es de justiciar social. }\end{array}$ & 20,212 & $-0,526$ & 0,089 & 0,911 & 0 \\
\hline $\begin{array}{l}\text { 26/11/2018- Estas son las listas de la co- } \\
\text { rrupción del PP y del PSOE. Los andalu- } \\
\text { ces estamos hartos. Contra la corrupción } \\
\text { no pueden luchar quienes no creen en la } \\
\text { limpieza de las instituciones. \#Deba- } \\
\text { teAndalucíaRTVE }\end{array}$ & 8,974 & $-0,526$ & 0,124 & 0,876 & 0 \\
\hline $\begin{array}{l}\text { 20/11/2018- Una nación decente no pro- } \\
\text { mote ni regala impunidad a quienes in- } \\
\text { tentan liquidar la democracia. Señores } \\
\text { del PSOE y de Podemos, ¿ustedes hu- } \\
\text { bieran indultado a Tejero? Nosotros } \\
\text { nunca. ¿Por qué quieren indultar a los } \\
\text { golpistas separatistas? \#STOPIndultos }\end{array}$ & 6,145 & $-0,373$ & 0,074 & 0,926 & \\
\hline
\end{tabular}


Tabla 19.(Continuación)

\begin{tabular}{|c|c|c|c|c|c|}
\hline $\begin{array}{l}\text { 18/11/2018- En Cs somos tan revolucio- } \\
\text { narios que no queremos ni que nos diri- } \\
\text { jan corruptos, ni que alguna personas } \\
\text { nombradas a dedo ocupen puestos de } \\
\text { direccióne n los servicios públicos por el } \\
\text { mero hecho de tener el carné del Partido } \\
\text { Socialista andaluz. \#AhoraSevilla }\end{array}$ & 4,507 & $-0,296$ & 0,052 & $-0,948$ & 0 \\
\hline $\begin{array}{l}\text { 19/11/2018- PP y PSOE nosh an hecho } \\
\text { creer durante } 40 \text { años que los andalu- } \\
\text { cesteníamos que conformarnos con las } \\
\text { peores tasas de paro y fracas escolar. } \\
\text { Pero los andaluces no nos resignamos: } \\
\text { este 2D temenos una gran oportunidad } \\
\text { para cambiar Andalucía. Ahorasí, vota- } \\
\text { Ciudadanos. \#DebateCanalSur }\end{array}$ & 4,457 & $-0,296$ & 0,049 & 0,952 & 0 \\
\hline
\end{tabular}

Elaboración propia a partir de datos de Twitter

\subsection{Adelante Andalucíal @AdelanteAND}

Adelante Andalucía registra un total de 115 tuits durante las jornadas previa, post y durante el debate de CanalSur ( $90 \%$ contenido relativo al propio debate o campaña), con actividad principal el día 19 de noviembre. Durante el debate de RTVE el número de tuits es menor (72), sobre todo durante el día de celebración, siendo $90 \%$ de las publicaciones relativas al contenido del debate. Los tuits restantes (sin contenido de campaña) tratan contenidos relacionados con el Día contra la Violencia de Género, así como el conflicto taxis/Uber.

En cuanto a los tuits con mayor engagement, predominan los relativos al segundo debate, así como del día previo. Atendiendo al tema principal, destacan las críticas hacia las fuerzas contrarias. En estos tuits predomina el sentiment con valor 0/nulo.

Tabla 20. Tuits primer debate perfil @AdelanteAND

\begin{tabular}{|l|c|c|c|}
\hline & $\begin{array}{c}\text { Jornada previa } \\
\mathbf{1 8 / 1 1 / 2 0 1 8}\end{array}$ & $\begin{array}{c}\text { Jornada debate } \\
\mathbf{1 9 / 1 1 / 2 0 1 9}\end{array}$ & $\begin{array}{c}\text { Jornada posterior } \\
\mathbf{2 0 / 1 1 / 2 0 1 8}\end{array}$ \\
\hline Tuits & 24 & 58 & 33 \\
\hline Tuits con contenido debate & 23 & 48 & 30 \\
\hline
\end{tabular}

Elaboración probia a partir de datos de Twitter 
Tabla 21. Tuits segundo debate perfil @AdelanteAND

\begin{tabular}{|l|c|c|c|}
\hline & $\begin{array}{c}\text { Jornada previa } \\
\mathbf{2 5 / 1 1 / 2 0 1 8}\end{array}$ & $\begin{array}{c}\text { Jornada debate } \\
\mathbf{2 6 / 1 1 / 2 0 1 9}\end{array}$ & $\begin{array}{c}\text { Jornada posterior } \\
\mathbf{2 7 / 1 1 / 2 0 1 8}\end{array}$ \\
\hline Tuits & 11 & 42 & 20 \\
\hline $\begin{array}{l}\text { Tuits con contenido de- } \\
\text { bate o campaña }\end{array}$ & 6 & 39 & 19 \\
\hline
\end{tabular}

Elaboración pronia a partir de datos de Twitter

Tabla 22. Tuits con mayor engagement en perfil @AdelanteAND y sentiment generado

\begin{tabular}{|c|c|c|c|c|c|}
\hline & Engage & $\begin{array}{l}\text { Com- } \\
\text { pound }\end{array}$ & $\begin{array}{c}\text { Nega- } \\
\text { tivo }\end{array}$ & $\begin{array}{c}\text { Neu- } \\
\text { tro }\end{array}$ & $\begin{array}{c}\text { Posi- } \\
\text { tivo }\end{array}$ \\
\hline $\begin{array}{l}\text { 26/11/2018- @TeresaRodr a Juanma Mo- } \\
\text { reno (PP) tras el \#DebateAndalucíaRTVE: } \\
\text { “Tú sabes que los Jedi defendían la Repú- } \\
\text { blica, no? }\end{array}$ & 40,08 & 0 & 0 & 1 & 0 \\
\hline $\begin{array}{l}\text { 19/11/2018- Juan Marín (C's): “Cataluña, } \\
\text { Cataluña..” ¿Y Andalucía pa'cuando, } \\
\text { Juan? }\end{array}$ & 41,297 & 0 & 0 & 1 & 0 \\
\hline $\begin{array}{l}25 / 11 / 2018 \text { - El que tiene que aprender } \\
\text { costumbres es Casado antes de cruzar } \\
\text { Despeñaperros. Aquí no queremos racism } \\
\text { y xenophobia mientras nosh anrobado a } \\
\text { manos llenas y Regalado millones de eu- } \\
\text { ros a la banca. Apocos kms, una embara- } \\
\text { zada perdía la vida en un naufragio, mal- } \\
\text { dito impresentable. }\end{array}$ & 27,78 & $-0,296$ & 0,051 & 0,949 & 0 \\
\hline $\begin{array}{l}\text { 25/11/2018- Es deleznable que con los ca- } \\
\text { davers aún calientes del ultimo naufra- } \\
\text { gio,Pablo Casado venga a decir que los } \\
\text { inmigrantes vienes a llevarse ayudas. El } \\
\text { rescate multimillonario a los bancos sí que } \\
\text { hace peligrar las ayudas sociales. @Tere- } \\
\text { saRodr }\end{array}$ & 23,839 & 0 & 0 & 1 & 0 \\
\hline
\end{tabular}


Tabla 22. (Continuación)

\begin{tabular}{|l|l|l|l|l|l|}
\hline $\begin{array}{l}\text { 26/11/2018- Deberíamos estar hablando } \\
\text { de educación y sanidad y el señir Juan } \\
\text { Marín (C's) ya ha vuelto a meter Cataluña } \\
\text { en el debate. }\end{array}$ & 16,364 & 0,34 & 0 & 0,909 & 0,091 \\
\hline
\end{tabular}

Elaboración propia a partir de datos de Twitter

\subsection{Teresa Rodríguezl @TeresaRodr_}

La candidata por Adelante Andalucía, Teresa Rodriguez, registra un total de 110 tuits durante las jornadas previa, post y durante el debate de CanalSur ( $90 \%$ contenido relativo al propio debate o campaña), con actividad principal los días 19 y 20 de noviembre. Durante el debate de RTVE, el número de tuits es menor (106), concentrándose la mayor actividad en el día de celebración, siendo $90 \%$ del contenido del debate.

Los tuits con mayor engagement se centran en la misma medida en ambos debates, siendo el máximo número alcanzado de engagement 3.21, con publicaciones en las que se personifica la problemática andaluza. En estos tuits predomina el sentiment con valor negativo de los usuarios.

Tabla 23. Tuits primer debate perfil @TeresaRodr_

\begin{tabular}{|l|c|c|c|}
\hline & $\begin{array}{c}\text { Jornada previa } \\
\mathbf{1 8 / 1 1 / 2 0 1 8}\end{array}$ & $\begin{array}{c}\text { Jornada debate } \\
\mathbf{1 9 / 1 1 / 2 0 1 9}\end{array}$ & $\begin{array}{c}\text { Jornada posterior } \\
\mathbf{2 0 / 1 1 / 2 0 1 8}\end{array}$ \\
\hline Tuits & 23 & 47 & 40 \\
\hline $\begin{array}{l}\text { Tuits con contenido } \\
\text { debate o campaña }\end{array}$ & 19 & 40 & 38 \\
\hline
\end{tabular}

Elaboración propia a partir de datos de Twitter

Tabla 24. Tuits segundo debate perfil @TeresaRodr_

\begin{tabular}{|l|c|c|c|}
\hline & $\begin{array}{c}\text { Jornada previa } \\
\mathbf{2 5 / 1 1 / 2 0 1 8}\end{array}$ & $\begin{array}{c}\text { Jornada debate } \\
\mathbf{2 6 / 1 1 / 2 0 1 9}\end{array}$ & $\begin{array}{c}\text { Jornada posterior } \\
\mathbf{2 7 / 1 1 / 2 0 1 8}\end{array}$ \\
\hline Tuits & 11 & 66 & 29 \\
\hline $\begin{array}{l}\text { Tuits con contenido } \\
\text { debate o campaña }\end{array}$ & 8 & 60 & 22 \\
\hline
\end{tabular}

Elaboración propia a partir de datos de Twitter 
Del mismo modo en la red personal de Teresa Rodríguez destacan los siguientes tuits:

Tabla 25. Tuits con mayor engagement en perfil @TeresaRodr_y sentiment generado

\begin{tabular}{|c|c|c|c|c|c|}
\hline & Engage & $\begin{array}{l}\text { Com- } \\
\text { pound }\end{array}$ & $\begin{array}{c}\text { Nega- } \\
\text { tivo }\end{array}$ & $\begin{array}{c}\text { Neu- } \\
\text { tro }\end{array}$ & $\begin{array}{c}\text { Posi- } \\
\text { tivo }\end{array}$ \\
\hline $\begin{array}{l}\text { 26/11/2018- Alicia, de } 65 \text { años, se ha sui- } \\
\text { cidado } \\
\text { tirándose del balcón cuando iba a ser } \\
\text { desahuciada. No es un suicidio, la han } \\
\text { asesinado la banca y los fondos buitres } \\
\text { que especulan con derechos básicos y } \\
\text { quienes una y otra vez que se niegan a re- } \\
\text { gular el Mercado de alquiler }\end{array}$ & 1,896 & 0,051 & 0,048 & 0,899 & 0,053 \\
\hline $\begin{array}{l}\text { 19/11/2018- Indignante que los fascistas } \\
\text { le } \\
\text { peguen palizas a feministas activistas en } \\
\text { las } \\
\text { calles con total impunidad. ¿Hasta cuándo } \\
\text { va a } \\
\text { seguir la ultra derecha hacienda apologia } \\
\text { del } \\
\text { fascismo en la calle y generando violencia } \\
\text { con el Estado hacienda oídos sordos? }\end{array}$ & 1,5 & 0 & 0 & 0,949 & 0 \\
\hline $\begin{array}{l}\text { 19/11/2018- Las madres andaluzas quere- } \\
\text { mos } \\
\text { que nuestros hijos e hijas vivan en una } \\
\text { Andalucía que les brined oportunidades, } \\
\text { derechos y Felicidad. \#MinutoDeOro } \\
\text { \#DebateCanalSur }\end{array}$ & 1,04 & 0 & 0 & 1 & 0 \\
\hline $\begin{array}{l}27 / 11 / 2018-\text { La creatividad andaluza no } \\
\text { tiene } \\
\text { límites. No lo he podido evitar, es que es } \\
\text { "de } \\
\text { age". }\end{array}$ & 0,907 & $-0,052$ & 0,227 & 0,773 & 0 \\
\hline
\end{tabular}

Elaboración propia a partir de datos de Twitter

\subsection{Vox/ @AndaluciaVox}

El perfil oficial del partido en Andalucía ha sido creado durante este año, por lo que no se registran tuits de campaña. En cuanto a su candidato, Francisco Serrano, sí presenta perfil, pero inactivo desde 2015, por lo que tampoco se puede hacer un seguimiento de campaña. 


\section{CONCLUSIONES}

Para realizar el análisis central de este artículo se han tomado como referencia las cuentas de Twitter de los partidos con representación parlamentaria en la Junta de Andalucía tras las elecciones celebradas el 2 de diciembre de 2018. La recogida de datos se ha basado en la actividad que tuvo lugar en los perfiles de los propios partidos y de sus candidatos durante los debates televisados del 19 y el 26 de noviembre, y los días previo y posterior a cada uno de ellos. De los datos extraídos del estudio se desprenden las siguientes conclusiones principales:

Atendiendo a la hipótesis planteada en la presente investigación, el empleo de Twitter como herramienta de partidos políticos y candidatos para conseguir la movilización de los votantes, así como la difusión de su ideología, podemos desprender de los resultados que se encuentra en niveles muy bajos. Esto se aprecia por un lado en la actividad de los perfiles (desigual tanto entre partidos, como entre partidos y sus candidatos) y por otro a través del engagement y el sentimiento generado entre las audiencias, que han ofrecido cifras reducidas, en el primer caso, y negativas, en el segundo. Si bien es cierto que existe actividad, se aprecia unidireccionalidad y no bidireccionalidad en la conversación, fundamental para lograr esa movilización.

En cuanto a los objetivos planteados, al analizar el OE1 se observa que el PSOE encabeza la lista como perfil con mayor actividad, seguido de Ciudadanos, PP y Adelante Andalucía. Esto no significa que exista correspondencia en la actividad de los perfiles de los candidatos: los perfiles de los partidos que encabezan la lista de mayor presencia en la red social Twitter, tienen a sus candidatos en el otro extremo (Susana Díaz no llega a la veintena de publicaciones relativas a campaña, y Juan Marín también presenta un número muy reducido). En el caso de la candidata por Adelante Andalucía, Teresa Rodríguez, se sitúa en el primer puesto en cuanto a actividad a pesar de que la presencia de su partido en esta red está a la cola. Con respecto a VOX, su actividad es inexistente.

Atendiendo al criterio de audiencia, no existe prácticamente interacción entre partidos y candidatos con los internautas; likes, shares y comments son escasos, por lo que el engagement (OE2) también lo es. Tan solo destaca el engagement de Adelante Andalucía y Juan Marín, debido a que la correlación entre el total de interacciones aumenta al encontrarnos con menor número de seguidores, mientras que los demás perfiles (con más seguidores) sitúan su ratio de engagement en baremos muy bajos, entre 0 y 1,5. Esto prueba que no existe correspondencia entre usuarios y comentarios. Precisamente estos comentarios tampoco aportan contenido que incite al debate, sino que muestran el recelo de los usuarios hacia los diferentes partidos/candidatos, o en su defecto, es aprovechado por algún militante para hacer propaganda, sin que llegue a destacar. 
Con respecto al OE3, el grado de atención otorgado por los diferentes perfiles a ambos debates, todos ellos tienen una actividad similar en cuanto a su dinámica de publicaciones, no sobresale un debate por encima de otro. Lo que sí se observa es que, en el caso de los partidos, PSOE y Ciudadanos, o en el de la candidata Teresa Rodríguez, tienen más presentes los debates en su estrategia de comunicación. Haciendo una comparativa entre partidos y candidatos también se constata que los perfiles de estos últimos presentan un menor número de publicaciones en las tres jornadas de muestra, mientras que su fuerza política correspondiente sí hace un uso más constante de esta red social y la actividad es considerablemente mayor.

Finalmente, dentro del OE4, relativo a las estrategias seguidas por los distintos partidos, el análisis confirma que utilizan Twitter como medio de ataque (para tratar de desvalorizar a rivales políticos), lo cual se observa fundamentalmente en los casos de PP y Adelante Andalucía, y como medio de difusión, área en la que PSOE y Ciudadanos destacan como las fuerzas que más se centran en sus candidatos, su ideología y/o en el propio debate. Atendiendo a los días seleccionados para este estudio, y como se indicaba anteriormente, queda clara la prioridad otorgada al debate en las cuentas de los partidos con respecto a las cuentas de los candidatos, con un perfil más electoralista las primeras, frente a las de las segundas, con un cariz más personal. Sin embargo, destaca en este análisis que los formatos electorales clásicos (debates televisados) siguen vigentes dentro de los nuevos formatos (redes sociales), atendiendo a su relevancia en la temática de las publicaciones.

Los datos muestran que la plataforma Twitter está decayendo en el ranking de relevancia a favor de otros formatos más recientes, provocando una migración de públicos. La falta de bidireccionalidad en la comunicación, deja entrever un uso cada vez menor del medio como entorno de conversación, representativo todavía pero actualmente en menor medida.

\section{Referencias}

Bruun, H. (2016). The Prism of Change: Continuity in Public ServiceTelevision in the Digital Era. Nordicom Review, 37(2): 1-17

Campos-Domínguez, E. (2017). Twitter y la ComunicaciónPolítica. ElProfesional de la Información. 26(5), pp. 785-793

Carrera-Fernández, M. J., Olmos, J. G., \&Peró, M. (2013). Psicología y lenguajeenpolítica: los candidatos a la Presidencia del Gobierno y suestilolingüístico. Anuario de psicología/The UB Journal of psychology, 43(1), 39-52

Casero-Ripollés, A. (2017). Producción de contenidospolíticos,empoderamientociudadano y públicosvulnerablesen la Web 2.0. EIProfesional de la Información, 26(1), pp. 13-19 
Ceron, A. \&D’Adda, G. (2015). E-campaigningon Twitter: Theeffectiveness of distributivepromises and negativecampaign in the 2013 Italianelection. New Media \&Society, 18, 1935-1955. doi:10.1177/1461444815571915

Chia-Shin, L. (2016). Convergence in electioncampaigns. Theframecontestbetween Facebook and mass media. International Journal of Researchinto New MediaTechnologies, $\mathrm{n}^{\circ} 22$, pp.199-214. doi: http://dx.doi.org/10.1177/1354856514545706

Congosto-Martínez, M. C. (2016). Caracterización de usuarios y propagación de mensajes en twitter en el entorno de temas sociales (Tesis Doctoral). Universidad Carlos III de Madrid

Congosto, M., Fernández, M. \& Moro, E. (2011). Twitter y política: Información, opinión y ¿predicción?. Cuadernos de Comunicación Evoca. 4. 11-15

Croft, W. \&Cruse, A. (2004), CognitiveLinguistics, Cambridge: Cambridge UniversityPress.

De la Fuente, J. R. (2010). Ciberpolítica, redes sociales y nuevasmovilizacionesenEspaña: el impacto digital en los procesos de deliberación y participaciónciudadana. Mediacionessociales, 7, 143-164.

Deltell, L., Congosto, ML., Claes, F. \& Osteso, JM.(2013): Identificación y análisis de los líderes de opinión en Twitter en torno a Hugo Chávez, en Revista Latina de Comunicación Social, 68, pp. 696 a 718. doi: 10.4185/RLCS-2013-997

Domínguez, D. C. (2009). Democracia 2.0: La política se introduce en las redes sociales. Democracy 2.0: politicsinside social networks. Pensar la publicidad, 3(2), 31

González, N. (2013). Métricas de la web social. Anuario ThinkEPI, 7, pp. 48-52

Grijelmo, Á. (2000). La seducción de las palabras (Vol. 2007). México: Taurus

Holtz-Bacha, C. (2013). Web 2.0: nuevos desafíos en comunicación política. Diálogo político, 30(1), 11-27

Horowitz, M.A. (2015). Public Service Media and Challegue of CrossingBorders: Assessing New models. MedijskeStudije, 6(12), pp. 80-90

IABSpain (2018). Estudioanual de redes sociales 2018. Madrid: Interactive Advertising Bureau

IABSpain (2019).Informe de TendenciasDigitales 2019. Madrid: Interactive Advertising Bureau

Kreiss, D. (2016). Seizing the moment: The presidential campaigns' use of Twitter during the 2012 electoral cycle. New media \& society, 18(8), 1473-1490

López Eire, A. \& y Guervós, JdS, (2000), Retórica y comunicación política. Madrid: Cátedra

López-García, G. (2016). Nuevos'y 'viejos' liderazgos: la campaña de las eleccionesgeneralesespañolas de 2015. Communication \& Society 29(3), 149-167 
Marcus, G. E. (2002). The Sentimental Citizen, Emotion in Democratic Politics. Pennsylvania: Pennsylvania State University Press

Marzal Felici. J. \& Zallo Elgezabal, R. (2016). Presentación: Las televisiones públicas de proximidad ante los retos de la sociedad digital. Communication\&Society 29(4), 1-7

Mazaira-Castro, A., Rúas-Araújo, J., Puentes-Rivera, I. (2019).Fact-Checking en los debates electorales televisados de las elecciones generales de 2015 y 2016. Revista Latina de Comunicación Social, 74, pp. 748 a 766

Moe, H. (2012). Who participates and how? Twitter as an arena for public debate about the Data Retention Directive in Norway. International journal of communication, v. 6, n. 1, pp. 12221244

Moya-Sánchez, M., \& Herrera-Damas, S. (2016). Cómo medir el potencial persuasivo en Twitter: propuesta metodológica. Palabra Clave, 19(3), 838-867

Orihuela, J.L. (2011). Mundo Twitter: una guía para comprender y dominar la plataforma que cambió la red. Barcelona: Alienta.

Rizo, M. (2003). Redes: Una aproximación al concepto. Recuperado de https://docplayer.es/10331781-Redes-una-aproximacion-al-concepto.html

Rodríguez-Andrés, R. \& Ureña-Uceda, D. (2011). Diez razones para el uso de Twitter como herramienta en la comunicación política y electoral (Ten reasons to use Twitter as a toolforpolitical and electoral communication). Comunicación y Pluralismo. 10. 89-116

Rúas-Araújo, J., Alves-Pérez, M. T., \& Fernández-Cabana, M. (2016). Comunicación, lenguaje y política: Análisis de los discursosinstitucionales del presidente de Ecuador, Rafael Correa (2007-2015), con la herramienta LIWC. Razón y Palabra, 20(95)

Small, G., \&Vorgan, G. (2009). El cerebro Digital. Cómo las nuevas tecnologías están cambiando nuestra mente.(19a. Edición, traducción de i-brain, 254). Barcelona: EdicionesUrano. Wright, R.(2000), Nonzero, The Logic of Human Destiny

Sobrino-Sande, J.C. (2018). Análisis de sentimientos en Twitter (TFM). Universidad Oberta de Catalunya

Stieglitz, S. \&Dang-Xuan, L. (2013). Emotions and InformationDiffusion in Social Media Sentiment of Microblogs and SharingBehavior. Journal of Management InformationSystems. 29. 217-248. 10.2753/MIS0742-1222290408

Trappel, J. (2016). Takingthepublicserviceremit forward acrossthe digital boundary. International Journal of Digital Television, 7(3), pp. 273-295

Tremblay, G. (2016). PublicService Media in theAge of Digital Networks.Canadian Journal of Communication, 41(4), pp. 191-206 
Trilling, D. (2015). Twodifferent debates? Investigatingtherelationshipbetween a political debate on TV and simultaneouscommentson Twitter. Social sciencecomputerreview, 33(3), 259276

Tumasjan, A. Oliver Sprenger, T., Sandner, P.\&Welpe, I. (2010). PredictingElectionswith Twitter: What 140 CharactersRevealaboutPoliticalSentiment. Word. Journal Of The International LinguisticAssociation. 10

Valerio, G., Herrera, D.J., Villanueva, F., Herrera, N. \& Rodríguez, M.C. (2015). Therelationshipbetween post formats and digital engagement: $A$ study of the Facebook pages of Mexicanuniversities. RUSC, 12(1), pp. 50-63

Zarrella, D. (2010). Can Twitter predictelections?.Recuperado de http://danzarrella.com/newdata-can-twitter-predict-elections.html 


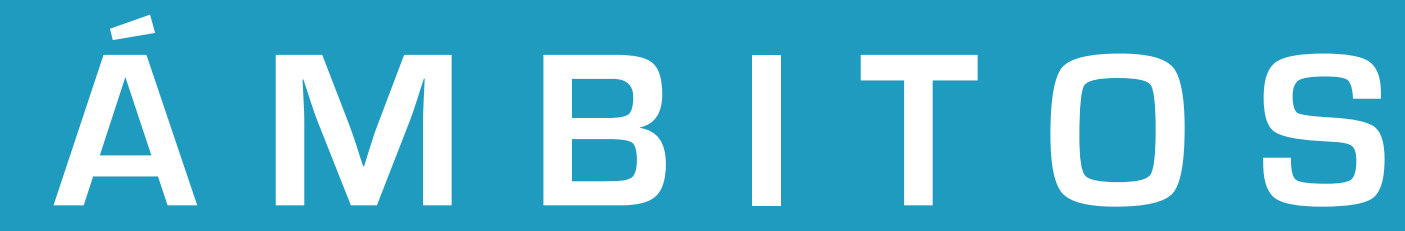

ISSN: 1139-1979 | ISSN digital: 1988-5733 | Depósito Legal: SE-1493-98

Revista Internacional de Comunicación editada por el Grupo de Investigación en Estructura, Historia y Contenidos de la Comunicación(GREHCCO) de la Universidad de Sevilla.

\section{ambitoscomunicacion@us.es}

http://institucional.us.es/ambitos

@RevistaAmbitos 